\title{
Length polymorphism in OGT between Korean native pig, Chinese Meishan, and the Western pig breeds
}

Yoon Seok Nam ${ }^{1+}$, Doo-Wan Kim ${ }^{2 \dagger}$, Myoung-Jik Kim², Kyu-Ho Cho ${ }^{2}$ and Jong Gug Kim ${ }^{1,3^{*}}$

\begin{abstract}
Background: The Korean native pig (KNP) is generally thought to have come from northern China to the Korean peninsula approximately 2000 years ago. KNP pigs were at the brink of extinction in the 1980s, since then efforts have been made to restore the breed by bringing together the remaining stocks in South Korea. As a result, KNP was registered as a breed in 2006. To find additional breed-specific markers that are distinct among pig breeds, variations in $\mathrm{O}$-linked $\mathrm{N}$-acetylglucosamine transferase (OGT) were investigated. OGT is located on chromosome $\mathrm{X}$ and catalyzes the post-translational addition of a single O-linked- $\beta$-N-acetylglucosamine to target proteins.
\end{abstract}

Findings: Length polymorphism in the intron 20 of OGT was identified. The intron 20 of OGT from Duroc, Landrace, and Yorkshire breeds was 281-bp longer than that from either KNP or Chinese Meishan pigs. The difference between the Western pig breeds (BB genotype) and KNP or Meishan pigs (AA genotype) was due to an inserted 276-bp element and the 5-bp ACTTG.

Conclusions: The polymorphism in OGT identified in this study may be used as an additional marker for determining the breed of origin among Meishan and the Western pig breeds. The length polymorphism suggests that the locus near OGT is not fixed in KNP. This marker would be relevant in determining the breed of origin in crossbred pigs between KNP pigs with known genotypes and the Western pig breeds with BB genotypes, thus confirming the contribution of the $X$ chromosome from each breed.

Keywords: O-linked N-acetylglucosamine transferase, OGT, Length polymorphism, KNP, Genotype

\section{Background}

The Korean native pig (KNP) is generally believed to have come from northern China to the Korean peninsula approximately 2000 years ago [1]. KNP pigs have black coat color in general and were at the brink of extinction in the 1980s due to their smaller animal size and slower growth rate [2]. Since then, effort has been made to restore the breed by bringing together the remaining stocks from a few provinces in the Republic of Korea [2-4]. As a result, the restored KNP has been registered as a breed in 2006 [3]. Genetic relationships between

\footnotetext{
* Correspondence: jonggugkim@jbnu.ac.kr

${ }^{\dagger}$ Equal contributors

'Department of Animal Sciences, College of Agriculture and Life Science, and Institute of Molecular Biology and Genetics, Chonbuk National

University, Jeonju-si, Jeollabuk-do 561-756, Republic of Korea

${ }^{3} 567$ Baekje-Daero, Deokjin-gu, Jeonju-si, Jeollabuk-do 561-756,

Republic of Korea

Full list of author information is available at the end of the article
}

KNP, Chinese Meishan [4] and Western [4,5] pig breeds have been determined using microsatellite markers. To find additional breed-specific markers that are distinct among pig breeds, several genes including O-linked $\mathrm{N}$ acetylglucosamine transferase (OGT) were investigated. $O G T$ is located on chromosome $\mathrm{X}$ and catalyzes the post-translational addition of a single O-linked- $\beta-\mathrm{N}$ acetylglucosamine (O-GlcNAc) on the hydroxyl groups of Ser and/or Thr residues of target proteins [6]. GlcNAcylation regulates cellular signaling and transcription processes in response to nutrients and stress, and has extensive crosstalk with phosphorylation [6]. OGT is also involved in nutrient sensing [7]. OGT has been mapped within the quantitative trait locus (QTL) affecting backfat depth, boar plasma FSH, and testicular weight using a length polymorphism within the intron 20 of OGT between Chinese and the Western pig breeds [8]. This genomic region located on chromosome $\mathrm{X}$ was 
investigated using Meishan (MS) $\mathrm{x}$ White composite (WC) crossbred boars [9]. Further, boars with MS alleles at the region had smaller testicles and lower total daily sperm production than boars with WC alleles [10]. In MS $x$ WC crossbred boars, the length polymorphism in $O G T$ was applicable to genotyping and identifying the breed of origin [8]. Therefore, the role of OGT and differential contribution of the $\mathrm{X}$ chromosome from each breed in crossbred pigs is worthy of further investigation. The first objective of this study was to determine whether the length polymorphism in the intron 20 of $O G T$ was present in KNP and this polymorphism could be used as a breed-specific marker among KNP, Chinese Meishan, and the Western pig breeds. In addition, KNP pigs have been used to produce specialty meat and they have been crossbred to Landrace or Yorkshire breeds for growth and meat quality trait studies in Korea [11-13]. Thus, the second objective of this study was to determine whether the length polymorphism can be used as a breed-specific marker in screening the breed of origin in crossbred pigs between KNP with known genotypes and the Western pig breeds.

\section{Methods}

\section{Experimental animals}

The experimental protocol and standard operating procedures on experimental animals were reviewed and approved by the Institutional Animal Care and Use Committee of the National Institute of Animal Science, RDA (Suwon, Republic of Korea), in compliance with standard international regulations. Initially, 10 animals for each breed from the breeding stocks at the National Institute of Animal Science, Rural Development Administration, Republic of Korea were analyzed for the presence of the length polymorphism. The animals included 10 Duroc sows, 5 Landrace boars and 5 sows, 3 Yorkshire boars and 7 sows, 5 KNP boars and 5 sows, 1 Meishan boar, 8 sows and 1 unknown sex, and 10 Duroc $x$ KNP crossbred sows. Subsequently, the number of pigs was increased to include 40 Duroc, 36 Landrace, 36 Yorkshire, 39 KNP, 10 Meishan, and 15 Duroc x KNP crossbred pigs $(n=176)$. Blood samples were collected and DNA was isolated.

\section{Primer design, PCR amplification, genotyping, and sequencing}

Primers were designed to amplify across the intron 20 based on the porcine OGT cDNA (GenBank accession no. DQ400859) and the gene sequence in the Genome Browser for pig (http://genome.ucsc.edu/). The forward (2968F) and reverse (3083R) primers correspond to bases 2968-2987 and 3083-3059 of the porcine OGT cDNA (GenBank accession no. DQ400859), respectively, and the expected size of the PCR amplicon across intron 20 was $631 \mathrm{bp}$. PCR reactions were carried out in a 20- $\mu \mathrm{l}$ volume containing $30 \mathrm{ng}$ of genomic DNA, $2 \mathrm{mM} \mathrm{MgCl}$, 10 pmol of each primer (OGT-2968F: 5' -GCACACCACA GGGATGGATG-3' and OGT- 3083R: 5' - GCTCAAGAC AACCTAAACAAGTAAG-3'), $200 \mu \mathrm{M}$ dNTP, and $2 \mathrm{U}$ Top-Ta $q^{\text {Tw }}$ DNA polymerase (Qiagen, Germany). Amplification was performed under the following PCR conditions: $10 \mathrm{~min}$ at $95^{\circ} \mathrm{C} ; 35$ cycles of $30 \mathrm{sec}$ at $95^{\circ} \mathrm{C}$, annealing for $30 \mathrm{sec}$ at $60^{\circ} \mathrm{C}$, and $1 \mathrm{~min}$ at $72^{\circ} \mathrm{C}$; and a final extension of $5 \mathrm{~min}$ at $72^{\circ} \mathrm{C}$. Length polymorphism among the pig breeds was determined after gel electrophoresis. Genotypes of the OGT gene among the Duroc, Landrace, Yorkshire, KNP, Meishan, and Duroc $x$ KNP pigs $(n=176)$ were determined. A number of pigs were sequenced to confirm the OGT genotypes of Duroc, KNP, and Meishan pigs.

\section{(A)}

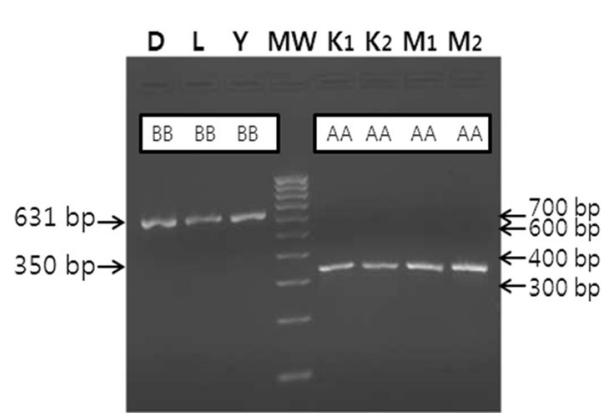

(B)
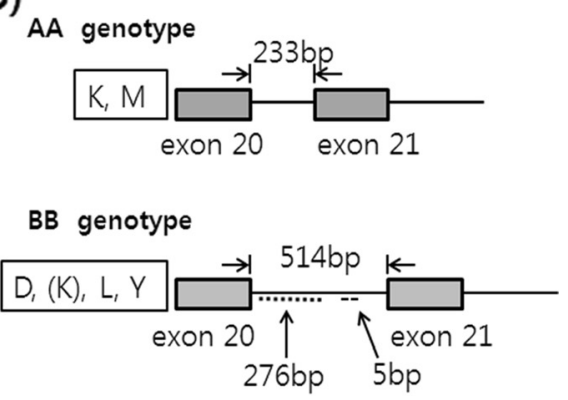

Figure 1 Genotypes of $O G T$ in different pig breeds and their schematic diagram. (A). The fragments containing the intron 20 of OGT from the Western pig breeds including Duroc $(D)$, Landrace $(L)$, and Yorkshire $(Y)$ with BB genotypes $(631 \mathrm{bp})$ were compared to those from Korean native pig (K) and Chinese Meishan (M) pigs with AA genotypes (350 bp). Single lane represents each breed for $D, L$, and $Y$ (lanes 1-3) and two lanes are included for $\mathrm{K}(\mathrm{K} 1$ and $\mathrm{K} 2$, lanes 5-6) and $\mathrm{M}$ (M1 and $\mathrm{M} 2$, lanes 7-8) with molecular weight (MW) marker. (B). Schematic diagram comparing the intron 20 of OGT between K, M (above) with AA genotypes and the Western (D, [K], L, and Y) (below) pig breeds with BB genotypes. The intron 20 of OGT from $K$ and $M$ was $233 \mathrm{bp}$, whereas that from D, (K), L and $Y$ was $514 \mathrm{bp}$. The longer intron 20 of OGT from the Western breeds contained an inserted 276-bp element (.....) near the beginning of the intron and the 5 bp-ACTTG ( - insertion in the middle of the intron. 


\section{Results and discussion}

Length polymorphism in the intron 20 of OGT among the pig breeds was determined by PCR and gel electrophoresis (Figure 1A). Both strands of the genomic DNA corresponding to the intron 20 of OGT were amplified from KNP, Duroc, and Meishan boars, sequenced, and then submitted to the GenBank (accession no. JQ045376-7 and JQ579450). Amplification of a fragment containing the intron 20 of $O G T$ by PCR revealed variations between KNP, Chinese Meishan and the Western pig breeds, including Duroc, Landrace, and Yorkshire. Fragments containing the intron 20 of $O G T$ appeared to be the same among Duroc, Landrace, and Yorkshire pigs and were longer than those from either Meishan or KNP pigs (Figure 1A). In the initial study, using 10 purebred pigs from each breed, amplicons from all 10 pigs from each breed were the same size, except KNP pigs (data not shown). Amplicons from 8 out of $10 \mathrm{KNP}$ pigs were the same, but those from the remaining two were different. The size of the fragment containing the intron 20 of OGT from Duroc boars was 631 bp (GenBank accession no. JQ045377), whereas those of Meishan (GenBank accession no. JQ579450) and 8 out of $10 \mathrm{KNP}$ pigs were $350 \mathrm{bp}$ (GenBank accession no. JQ045376). Sequencing indicated that the sizes of OGT intron 20 of the Duroc boars were $514 \mathrm{bp}$, while those from Meishan or KNP boars were $233 \mathrm{bp}$, resulting in a difference of $281 \mathrm{bp}$. The size and sequence of the intron 20 OGT from Duroc boars were identical to the one in the Genome Browser for pig (http://genome.ucsc.edu/). The 281-bp difference in the intron between Duroc and Meishan or KNP was due to an inserted 276-bp element near the beginning of the intron and the 5-bp ACTTG insertion in the middle of the intron (Figure 1B). These results are in agreement with a previous study on Meishan and White composite pigs. In that study, using 83 boars of 16 litters from inter se matings of $1 / 2$ Meishan (MS) $\times 1 / 2$ White composite (WC) parents, the breeds of origin of $\mathrm{X}$ chromosome QTL were confirmed based on this OGT polymorphism in the boars. The boars had either MS allele with AA genotype or WC allele with BB genotype (Kim JG, Nonneman D, Rohrer GA, unpublished observations).

When the entire intron 20 sequence of $O G T$ from Duroc (GenBank accession no. JQ045376) was compared using the algorithm "Basic Local Alignment Search Tool (BLAST)", it was revealed that several homologous regions to this sequence existed in the human chromosome $\mathrm{X}$ and in pig chromosomes. However, when the inserted 276-bp element of the intron 20 was compared using the BLAST, homologous regions existed only in several pig chromosomes, but not in any other species. The sizes of the OGT intron 20 from human, cattle, and mice were 240, 243, and 262 bp (http://genome.ucsc. $\mathrm{edu} /$ ), respectively, and thus, they are similar to the 233bp intron from either Meishan or KNP boars. Sequence homologies of the OGT intron 20 of Meishan and KNP boars, which do not contain the inserted 276-bp element and the 5-bp ACTTG of the OGT intron 20, with those of the human, cattle and mice were $76.3 \%, 69.5 \%$, and $62.0 \%$, respectively. This suggests that the 276-bp element and the 5-bp ACTTG of the OGT intron 20 in Duroc and other Western pig breeds may have been inserted differentially during the domestication process in different regions.

There was a variation in the intron 20 OGT among the $10 \mathrm{KNP}$ pigs in the initial study. Unexpectedly, 8 out of the $10 \mathrm{KNP}$ pigs had a short intron, which is the same as the Meishan pigs, whereas $2 \mathrm{KNP}$ pigs had a long intron, which is the same as the Western pigs (data not shown). When additional pigs were analyzed, KNP pigs had short and long intron 20 of $O G T$, and others were

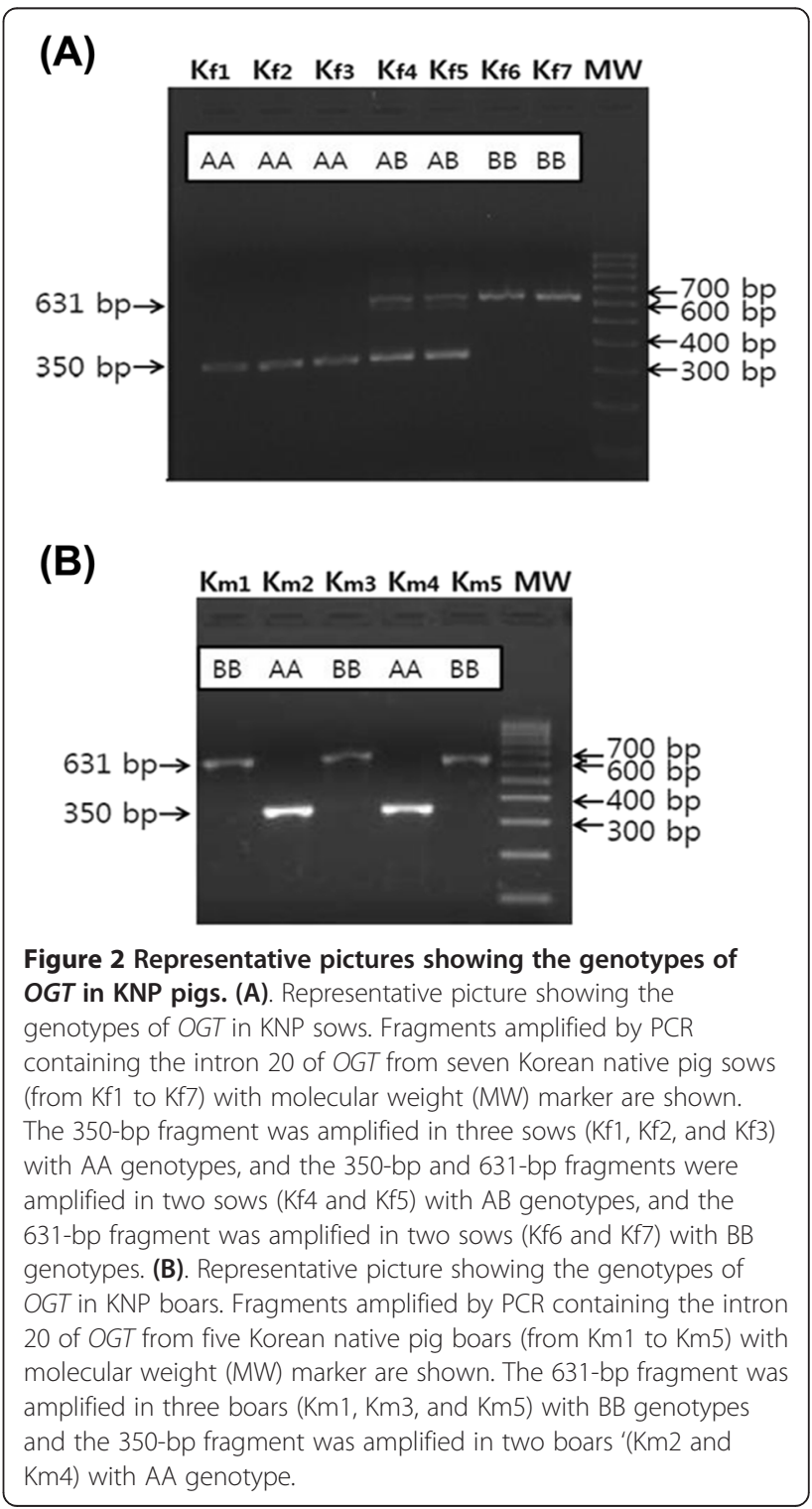


Table 1 Frequency of OGT genotypes among different pig breeds

\begin{tabular}{lllll}
\hline & AA & AB & BB & Total \\
\hline Duroc & 0 & 0 & 40 & 40 \\
Landrace & 0 & 0 & 36 & 36 \\
Yorkshire & 0 & 0 & 36 & 36 \\
KNP & 16 & 13 & 10 & 39 \\
KNP boar) $^{\mathrm{a}}$ & $(7)$ & $(0)$ & $(3)$ & $(10)$ \\
(KNP sow) $^{\mathrm{b}}$ & $(9)$ & $(13)$ & $(7)$ & $(29)$ \\
Meishan & 10 & 0 & 0 & 10 \\
Duroc $\times$ KNP & 0 & 2 & 13 & 15 \\
Total & 28 & 13 & 135 & 176 \\
\hline
\end{tabular}

a, $\mathrm{b}$ Boars and sows of KNP pigs were calculated within ( ).

heterozygotes, and thus the genotypes were designated as $\mathrm{AA}, \mathrm{BB}$, and $\mathrm{AB}$, respectively (Figure $2 \mathrm{~A}, \mathrm{~B}$ ). In contrast to the KNP pigs, the genotypes of OGT in Meishan pigs and the Western breeds were fixed as $\mathrm{AA}$ and $\mathrm{BB}$, respectively (Table 1 ). When genotypes of $39 \mathrm{KNP}$ pigs were analyzed, there were 16 AA (41.0\%), $13 \mathrm{AB}$ (33.3\%), and $10 \mathrm{BB}(25.6 \%)$. When we compared the genotypes of OGT between KNP pigs born in 2009 and in 2010 or 2011, the frequency of allele A decreased slightly in 2010 or 2011 in comparison to 2009. The genotypes of 18 pigs born in 2009 were 8 AA (44.4\%), $6 \mathrm{AB}$ (33.3\%), and $4 \mathrm{BB}(22.2 \%)$. However, the genotypes of 21 pigs born in 2010 or 2011 were $8 \mathrm{AA}$ (38.1\%), $7 \mathrm{AB}$ (33.3\%), and $6 \mathrm{BB}(28.6 \%)$. The results suggest that the locus is not fixed near OGT in KNP pigs. The observed frequency reduction of genotype AA among KNP pigs born in 2011 or 2012 could be due to the sampling bias of including more sows born from parents of $\mathrm{AB}$ or $\mathrm{BB}$ genotypes (Table 1). However, we cannot rule out that the reduction is due to the heterogametic state of boars

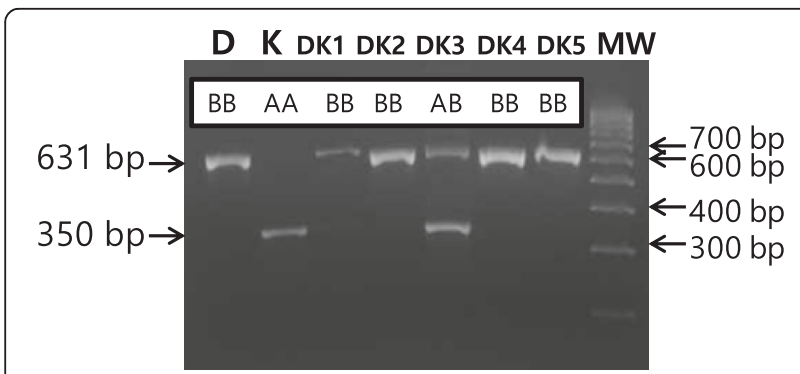

Figure 3 Genotypes of OGT from D, K, and D x K crossbred sows. After two generations of crossbreeding between Duroc (D) and Korean native pig $(K)$, the genotypes of four out of five Duroc $x$ Korean native pig crossbred (DK) sows (DK1, 2, 4, and 5 in lanes 3, 4, 6 and 7) were BB, having the same 631-bp fragment amplified as D. The genotype of one (DK3 in lane 5) of the five DK sows was $A B$, having both the $631 \mathrm{bp}$ fragment, which is the same as the Duroc sow (in lane 1) and the 350-bp fragment, which is the same as the KNP sow (in lane 2). or the result of genetic drift in males. After two generations of crossbreeding between Duroc with KNP pigs, all five Duroc x KNP (DK) crossbred boars and eight of 10 DK crossbred sows had BB genotypes, which is the same as Duroc boars, while the remaining two DK crossbred sows had AB genotypes (Table 1) (Figure 3).

\section{Conclusions}

In conclusion, the length polymorphism in the intron 20 of OGT may be used as an additional marker for determining the breed of origin among Chinese Meishan and the Western pig breeds including Duroc, Landrace, and Yorkshire. The polymorphism identified in this study suggests that the locus near OGT is not fixed in KNP pigs, and this marker may supplement the restoration effort of KNP as an additional mean to verify the origin of the breed near this locus. It would be also relevant in determining the breed of origin in crossbred pigs between KNP with known genotypes and Duroc or other Western breeds with $\mathrm{BB}$ genotypes, and thus confirming the contribution of the $\mathrm{X}$ chromosome from each breed.

\section{Competing interests}

The authors declare that they have no competing interests.

\section{Authors' contributions}

YSN performed DNA isolation and OGT fragment amplification by PCR. DWK designed breeding experiments, performed the breeding of pigs and collected the blood. MJK designed the breeding experiments and helped to draft the manuscript. KHC designed the breeding experiments and helped to draft the manuscript. JGK conceived of the study, participated in its design, analyzed the polymorphism, and wrote the draft. All authors read and approved the final manuscript.

\section{Acknowledgements}

This work was supported in part by a grant from the Next-Generation BioGreen 21 Program (No. PJ008047), Rural Development Administration, Republic of Korea.

\section{Author details}

${ }^{1}$ Department of Animal Sciences, College of Agriculture and Life Science, and Institute of Molecular Biology and Genetics, Chonbuk National University, Jeonju-si, Jeollabuk-do 561-756, Republic of Korea. ${ }^{2}$ Swine Science Division, National Institute of Animal Science, RDA, 114 Sinbang 1-gil, Seonghwan-eup, Seobuk-gu, Cheonan-si, Chungnam 331-801, Republic of Korea. ${ }^{3} 567$ Baekje-Daero, Deokjin-gu, Jeonju-si, Jeollabuk-do 561-756, Republic of Korea.

Received: 30 August 2014 Accepted: 20 January 2015 Published online: 14 March 2015

\section{References}

1. Kim KS, Choi CB. Genetic structure of Korean native pig using microsatellite markers. Korean J Genet. 2002;24:1-7.

2. Kwon OS. Preservation and current status of Korean native pig-in Korean. In: Lee SJ et al., editors. Symposium on preservation and utilization of Korean native pig-in Korean. Chonan, Republic of Korea: Sangrok-sa; 2006. p. 3-20.

3. Kim MJ. Restoration of Korean native pig-in Korean. In: Jung IB, editor. Raising Korean native pig-in Korean. Chonan, Republic of Korea: Sangrok-sa; 2008. p. 21-30.

4. Kim TH, Kim KS, Choi BH, Yoon DH, Jang GW, Lee KT, et al. Genetic structure of pig breeds from Korea and China using microsatellite loci analysis. J Anim Sci. 2005;83:2255-63.

5. Kim MJ, Li GH, Oh JD, Cho KH, Jeon GJ, Choi BH, et al. Characterization of a Korean traditional porcine breed using microsatellite markers and the 
establishment of an individual identification system. Korean J Food Sci Anim Resour. 2007;27:150-6.

6. Butkinaree C, Park K, Hart GW. O-linked beta-N-acetylglucosamine (O-GICNAc): Extensive crosstalk with phosphorylation to regulate signaling and transcription in response to nutrients and stress. Biochim Biophys Acta. 1800:2010:96-106

7. Hanover JA, Krause MW, Love DC. The hexosamine signaling pathway: O-GIcNAc cycling in feast or famine. Biochim Biophys Acta. 1800;2010:80-95

8. Kim JG, Ford JJ, Rohrer GA, Nonneman D. Molecular cloning of porcine OGT cDNA and mapping to the X chromosome [abstract]. Biol Reprod. 2006; Supplement:154.

9. Rohrer GA, Wise TH, Lunstra DD, Ford JJ. Identification of genomic regions controlling plasma FSH concentrations in Meishan-White Composite boars. Physiol Genomics. 2001;6:145-51.

10. Ford JJ, Wise TH, Lunstra DD, Rohrer GA. Interrelationships of porcine $X$ and $Y$ chromosomes with pituitary gonadotropins and testicular size. Biol Reprod. 2001;65:906-12.

11. Kim EH, Choi BH, Kim KS, Lee CK, Cho BW, Kim TH, et al. Detection of Mendelian and parent-of-origin quantitative trait loci in a cross between Korean native pig and Landrace I. Growth and body composition traits. Asian Austral J Anim. 2007;20:669-76.

12. Kim SW, Li XP, Lee YM, Choi YY, Cho BW, Choi BH, et al. QTL scan for meat quality traits using high-density SNP chip analysis in cross between Korean native pig and Yorkshire. Asian Austral J Anim. 2011;24:1184-91.

13. Niu P, Kim SW, Choi BH, Kim TH, Kim JJ, Kim KS. Porcine insulin-like growth factor 1 (IGF1) gene polymorphisms are associated with body size variation. Genes Genom. 2013;35:523-8.

\section{Submit your next manuscript to BioMed Central and take full advantage of:}

- Convenient online submission

- Thorough peer review

- No space constraints or color figure charges

- Immediate publication on acceptance

- Inclusion in PubMed, CAS, Scopus and Google Scholar

- Research which is freely available for redistribution 